\title{
Wild food plants and wild edible fungi of Heihe valley (Qinling Mountains, Shaanxi, central China): herbophilia and indifference to fruits and mushrooms
}

\author{
Yongxiang Kang ${ }^{1}$, Łukasz Łuczaj ${ }^{2 *}$, Sebastian Ye3 , Shijiao Zhang ${ }^{1}$, Jin Kang ${ }^{1}$ \\ ${ }^{1}$ College of Forestry, Northwest A\&F University, Yangling 712100, China \\ ${ }_{2}^{2}$ Department of Botany and Biotechnology of Economic Plants, University of Rzeszów, Werynia 502, 36-100 Kolbuszowa, Poland \\ ${ }^{3}$ Skargi 11/70, 39-300 Mielec, Poland
}

\begin{abstract}
The aim of the study was to investigate knowledge and use of wild food plants and fungi in Han (i.e. Chinese) nationality villages in central China, including famine plants used in the respondents' childhood. A valley adjacent to the extremely species-rich temperate forest vegetation of the Taibai Nature Reserve was chosen. Eighty-two people from 5 villages took part in the study. Altogether, 159 wild food plant species and 13 fungi folk taxa were mentioned by informants. The mean number of freelisted wild foods was very high (24.8; median - 21.5). An average respondent listed many species of wild vegetables (mean - 17, median - 14.5), a few wild fruits (mean - 5.9 and median - 6) and very few fungi (mean - 1.9, median - 1), which they had eaten.

Over $50 \%$ of respondents mentioned gathering the young shoots or leaves of Celastrus orbiculatus, Staphylea bumalda and S. holocapra, Caryopteris divaricata, Helwingia japonica, Pteridium aquilinum, Pimpinella sp., Amaranthus spp., Matteucia struthiopteris, Allium spp., Cardamine macrophylla and Chenopodium album. Only one species of fruits (Schisandra sphenanthera) and none of the mushrooms were mentioned by over half of the respondents. Although very diverse, it can be noted that the use of wild vegetables has decreased compared to the second half of the 20th century, as informants listed several plants which they had stopped using (e.g. Abelia engleriana) due to the availability of cultivated vegetables and other foodstuffs. On the other hand, the collection of the most well-known wild vegetables is maintained by selling them to tourists visiting agritourist farms, and restaurants.
\end{abstract}

Keywords: ethnobotany, ethnomycology, wild edible plants, non-timber forest products, mycophobia, food security

\section{Introduction}

Wild food plant and fungi use in the world is very diverse. In many areas, mainly in urban centers or in the richest countries, few wild species are used, usually just a few wild fruits, mushrooms or green vegetables. However, in less developed countries wild food is still a significant component of nutrition. The use of wild greens and fungi shows a particularly interesting pattern as in some areas of the world these components of the diet are either avoided or little used (herbophobia and mycophobia, respectively), whereas in other areas they constitute an everyday part of nutrition [1]. For example in Amazonia and Eastern Europe wild greens are little used, in contrast to East Asia, India and parts of Africa [2]. Wild mushrooms are widely gathered in Italy, France, Catalonia, Slavic countries,

*Corresponding author. Email: lukasz.luczaj@interia.pl

This is an Open Access digital version of the article distributed under the terms of the Creative Commons Attribution 3.0 License (creativecommons.org/licenses/by/3.0/), which permits redistribution, commercial and non-commercial, provided that the article is properly cited.
Turkey, Mexico and parts of Africa, but traditionally little used in England [3].

The country the most renowned for its wide contemporary use of wild components in the human diet is China. This stems from a few factors $[4,5]$ :

(i) China frequently suffered severe food crises up until the 1960 s, so the use of any natural resources was important to the inhabitants,

(ii) wild plants and animals are believed to contain more "qi" ("life energy"), so they are regarded as more nutritious and healthy,

(iii) Chinese culinary art likes indulging in a large number of, often strange, ingredients.

Although the research on potentially edible wild plants has been well developed in China (e.g. [5-10]), studies focused on documenting local traditions of wild plant and fungi use using the methodology of modern ethnobotany are relatively few, and the publications in international papers solely concern ethnic minorities, i.e. Mongolians [11,12], Shaxi in Sichuan [13,14], Miao in Hunan [15] and a variety of ethnic groups in Yunnan [9,10,16-18]. Two papers (in Chinese) concerning wild food resources in the Qinling Mounatins were published, but from a different part of these mountains [19,20]. The relatively short list of species given in them $[19,20]$ suggests that only the most 
commonly used species were included. The ethnomycology of edible mushrooms in China is also under-developed (in spite of the extensive literature on the economic use of mushrooms in China), and particularly lacking are studies simultaneously oriented towards wild vegetables, mushrooms and fruits.

Paradoxically, little is known about wild food plant use patterns in north-central, central and eastern China where the dominant Han (i.e. "Chinese") population lives. The aim of our study was to fill this gap and to record wild food species in one little-developed, mountainous, rural area in the province of Shaanxi. For our study we chose probably the best-preserved forest complex in central China - the vicinity of the Taibai Nature Reserve, where the local population has a particularly rich choice of wild food plants and mushrooms.

The working hypothesis was that in an area of high biodiversity, in a country famous for the use of many species of wild foods, the farmers use a large number of wild vegetables, fruits and mushrooms in their nutrition, and this knowledge is widespread in the community. An additional aim was to elicit answers on the differences between present day use and use during the last widespread famine (1958-1960).

\section{Study area}

The study was located in the Heihe National Forest Park, on the southern edge of the Taibai Nature Reserve, with the highest peak of northern China in the center of the reserve (Mt Taibai $3767 \mathrm{~m}$ a.s.l.). The nature reserve protects a highly diverse flora - from warm temperate (with subtropical elements) to alpine at the top. The National Forest Park (a less strict protection regime) is the southern extension of it, and mainly protects species-rich forests. The area is completely covered by ancient forest vegetation and rocky outcrops. The river Heihe valley belongs to the Houzhenzi administrative unit [town, zhen (镇)], with an area of $822 \mathrm{~km}^{2}$. It is a very isolated place, which has vehicular access to the county town of Zhouzhi (where the post-office and schools are located) only via a $2.5 \mathrm{~h}$ drive through a winding precipitous gorge, often blocked for days by falling rocks. The whole valley is inhabited by 3500 people - ca. a thousand in the main settlement of Houzhenzi, and the rest in the hamlets scattered in the forest (Fig. 1).

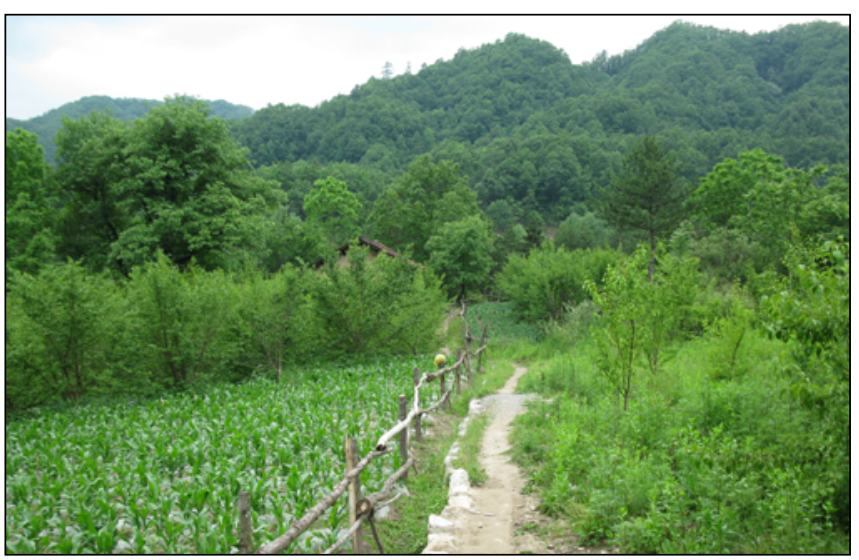

Fig. 1 A typical landscape in Houzhenzi valley - small fields in a valley surrounded by dense primary forest vegetation. Photograph by Łukasz Łuczaj.

The studied villages lie between 1000 and 1400 a.s.l. At these altitudes the climate is humid temperate, with daily temperatures in summer oscillating around $20-30^{\circ} \mathrm{C}$ and winter temperatures around $10^{\circ} \mathrm{C}$ to $-10^{\circ} \mathrm{C}$. The mean annual temperature in Houzhenzi is $8.2^{\circ} \mathrm{C}$, with high rainfall of nearly $1000 \mathrm{~mm}$, out of which $44 \%$ is concentrated in the summer months [21]. The dominant vegetation is the species-rich Quercus variabilis and Q. aliena var. acuteserrata forest, with an admixture of Pinus tabulaeformis, and many deciduous tree species (e.g. Acer spp., Tilia spp.).

The majority of the local population are subsistence farmers who grow maize, potatoes, wheat and beans [21,22]. Sources of cash income are the orchards of zaopi (Cornus officinalis), walnuts (Juglans regia) and northern Sechuan pepper (Zanthoxylum bungeanum). Digging out medicinal roots and collecting medicinal herbs for wholesale buyers is also a very popular activity $[21,22]$.

\section{Methods}

The field research was conducted in June and July 2011, as well as in August 2012, using transect walks and semistructured interviews with key informants, individual and group freelisting interviews (36 freelists were created), and cross-checking of the gathered herbarium specimens with key informants. Altogether, 82 people from five settlements (Houzhenzi, Diaoyutai, Huaerping, Jiangjiaping, Sanhe) took part in the study. The mean age of participants was 50 (from 16 to 83 ). The data were supplemented by participant observations by one of the authors (S. Y.) who had frequently visited the area, in 2007-2010, during all seasons of the year. During freelisting we separately asked, which species of wild vegetables (including underground organs), wild fruits and wild mushrooms were used. Making the three separate freelists enabled the comparison of the use of these categories and helped elicit answers from the respondents [23,24]. Freelists were made orally and written down on the spot by our team, including the Chinese-script version of the plant/fungi names.

The nine restaurants selling wild vegetables in Houzhenzi were also visited and menus were photographed in order to record the taxa sold and their price.

The study started from a few informants found using the snowball technique, but most interviewees were found by systematic walks through the village, visiting houses and asking the inhabitants if they wanted to take part in the study. We aimed at interviewing only one person from each household, only occasionally were two people from the same house interviewed, if there were signs that their knowledge differed (e.g. one of the spouses comes from another village, etc.). We also displayed some of our collections (mushrooms and some wild vegetables) in the main street of Houzhenzi, to observe the reactions and comments of people to the edible taxa shown.

Voucher specimens were collected from the specimens gathered during transect walks or supplied by informants, and are stored in the Department of Forestry, Northwest A\&F University in Yangling.

\section{Results}

\section{General figures}

Altogether, 159 plant species from 59 families (classified as 139 folk taxa) and 13 fungi taxa were mentioned by the informants as eaten at least once, but only 128 plant species 
and 12 fungi species were confirmed as eaten by more than one person (Tab. 1, Tab. 2, Tab. 3). The leaves and green parts of 104 species have been used, roots/rhizomes/tubers/bulbs of 18 species , flowers of 4 species and fruits of 36 species. Respondents mentioned wild vegetables most eagerly, and generally had problems listing wild fruits and fungi, stating that they are unimportant and are collected rarely. The gathering of young shoots of Celastrus orbiculatus and Staphylea spp. (mainly Staphylea bumalda, also S. holocarpa) was mentioned by nearly every respondent. Over $50 \%$ of respondents also mentioned gathering the young shoots or leaves of Caryopteris divaricata, Helwingia japonica, Pteridium aquilinum, Pimpinella sp., Amaranthus spp., Matteucia struthiopteris, Allium spp., Cardamine macrophylla and Chenopodium album (Fig. 2, Fig. 3). Six wild vegetables are served in most local restaurants (Tab. 3). Only one species of fruits (Schisandra sphenanthera) and none of the mushrooms were mentioned by over half of the respondents.

The mean number of freelisted wild foods was 24.8 (median - 21.5). An average respondent listed many species of wild vegetables (mean - 17, median - 14.5), a few wild fruits (mean -5.9 and median -6 ) and very few fungi (mean - 1.9, median -1$)$ as eaten.

The domination of wild vegetables in foraging activities is also confirmed by the fact that they are the only category of wild food stored for winter. Drying is a very common preserving technique (Fig. 4). Households dry between 1-5 species each year, usually a few kg of dry shoots and leaves, but some households who host tourists [so called “nong jia le” (农家乐)] can even dry a few dozen kg of dry "ye cai" (wild vegetables). Particularly large amounts of Chenopodium album are dried, as they are often used as winter fodder for pigs as well. Other commonly dried foods include Staphylea spp., Helwingia japonica, Celastrus orbiculatus, Toona sinensis and Cardamine macrophylla. Also, plants which are usually not treated as "ye cai", but as pig food (e.g. Artemisia subdigitata), are dried for the animals. In 2011 and 2012 dried Staphylea shoots were sold at the local food shop in Houzhenzi at $40 ¥ / \mathrm{kg}$. Dried Chenopodium was, in 2011, sold to tourists a few $\mathrm{km}$ before entering the village, along with dried Auricularia and Lentinula mushrooms. Most families dry 1-5 species of wild vegetables outside on the concrete, on mats or inside the house on newspapers. Formerly wild vegetables were lacto-fermented, but now this is done very rarely.

Forest species, species of grasslands and forest edges as well as ruderal species are well represented in the list of collected taxa (however typical forest species dominate with $44 \%$ taxa, only $15 \%$ taxa are ruderal species, the rest are ubiquitous species or species of intermediate successional stages). The ruderal species are collected near homesteads. Their growth is often promoted. For instance when a farmer sprays their Cornus officinalis plantation with glyphosphate, they leave a clump of wild vegetables unsprayed. One of the most protected species is Chenopodium album, which is harvested and dried at the turn of May and June. Some forest species are harvested up to $5 \mathrm{~km}$ from the villages, up to the altitude of $1800 \mathrm{~m}$ a.s.l. At even higher altitudes, wild plants are only harvested while collecting medicinal herbs, which grow even higher.

All the older informants were asked about plants eaten during the severe food shortages that plagued China until the last case of famine in 1958-1960. The usual response was that they ate the same species but in larger quantities. They said that they were lucky having so many wild vegetables around, as some people from other, more populated areas had to take refuge in
Tab. 1 Most commonly freelisted species in the study.

\begin{tabular}{|c|c|c|}
\hline Species & Category & $N=36$ \\
\hline Celastrus orbiculatus & $\mathrm{v}$ & 35 \\
\hline Staphylea spp. & $\mathrm{v}$ & 33 \\
\hline Caryopteris divaricata & $\mathrm{v}$ & 27 \\
\hline Helwingia japonica & $\mathrm{v}$ & 27 \\
\hline Pteridium aquilinum & $\mathrm{v}$ & 26 \\
\hline Pimpinella sp. & $\mathrm{v}$ & 24 \\
\hline Amaranthus spp. & $\mathrm{v}$ & 24 \\
\hline Matteucia struthiopteris & $\mathrm{v}$ & 23 \\
\hline Cardamine macrophylla & $\mathrm{v}$ & 22 \\
\hline Schisandra sphenanthera & $\mathrm{f}$ & 22 \\
\hline Chenopodium album & $\mathrm{v}$ & 22 \\
\hline Allium spp. & $\mathrm{v}$ & 20 \\
\hline Toona sinensis & $\mathrm{v}$ & 18 \\
\hline Akebia trifoliata & $\mathrm{f}$ & 18 \\
\hline Rubus spp. & $\mathrm{f}$ & 17 \\
\hline Prunus salicina & $\mathrm{f}$ & 15 \\
\hline Chrysosplenium biondianum & $\mathrm{v}$ & 15 \\
\hline Saussurea dolichopoda & $\mathrm{v}$ & 14 \\
\hline Decaisnea fargesii & $\mathrm{f}$ & 14 \\
\hline Adenophora spp. & $\mathrm{v}$ & 13 \\
\hline Cantharellus cibarius & $\mathrm{m}$ & 13 \\
\hline Allium paepalanthoides & $\mathrm{v}$ & 12 \\
\hline Sedum amplibracteatum & $\mathrm{v}$ & 11 \\
\hline Eleagnus umbellata & $\mathrm{f}$ & 11 \\
\hline Tricyrtis macropoda & $\mathrm{v}$ & 11 \\
\hline
\end{tabular}

$\mathrm{f}$ - fruit; $\mathrm{m}$ - mushroom; $N$ - number of freelists; $\mathrm{v}$ - green vegetable.

Tab. 2 Wild food plants sold in the restaurants in Houzhenzi.

\begin{tabular}{ll}
\hline \multicolumn{1}{c}{ Species } & N \\
\hline Staphylea spp. (mainly S. bumalda) & 9 \\
Toona sinensis & 8 \\
Chenopodium album & 7 \\
Matteucia struthiopteris & 6 \\
Pteridium aquilinum & 6 \\
Celastrus orbiculatus & 6 \\
Helwingia japonica & 2 \\
Medicago sativa & 1 \\
Cardamine macrophylla & 1 \\
Caryopteris divaricata & 1 \\
Pimpinella sp. & 1 \\
Lychnis senno & 1 \\
\hline
\end{tabular}

$N$ - No. of restaurants selling wild food plants $($ total $=9)$.

their mountains to avoid starvation. However, asking questions about the last time the particular species was used revealed that there is a group of taxa which could clearly be called famine plants - species, which were used in this area until the mid20th century and are not used any more. These include: the leaves of Abelia engleriana, the rhizomes of Pueraria lobata, 
Tab. 3 The list of wild edible plants and fungi confirmed by at least two respondents.

Family (according to

Scientific name
APGIII [31])
Local name in

Part used

Frequency Local name in pinyin

Chinese

\section{Vascular plants}

Abelia engleriana Rehder

Achyranthes bidentata Blume

Actinidia chinensis Planch.

Adenophora spp. (A. capillaris Hemsl., A. polyantha Nakai)

Akebia trifoliata (Thunb.) Koidz.

Allium ovalifolium Hand.-Mazz., A. cf. victorialis L.

Allium paepalanthoides Airy Shaw

Allium spp. (A. cf. senescens L., A. macrostemon Bunge)

Amaranthus caudatus L.

Amaranthus retroflexus L., A. paniculatus L., A. viridis $\mathrm{L}$.

Anaphalis aureopunctata Lingelsh et Borza

Anaphalis margaritacea Benth. \& Hook. f.

Aralia chinensis L.

Arctium lappa L.

Artemisia subdigitata Mattf.

Asarum himalaicum Hook. f. \& Thomson ex

Klotzsch

Asarum sieboldii Miq.

Begonia sinensis A. DC.

Berchemia sinica Schneid.

Boehmeria gracilis C. H. Wright

Boehmeria tricuspis Makino

Broussonetia papyrifera (L.) Vent.

Cacalia roborowskii (Maxim.) Y. Ling

Capsella bursa-pastoris Medik.

Cardamine macrophylla Willd.

Cardamine spp. (other smaller species e.g. C.

flexuosa With., C. hirsuta L.)

Caryopteris divaricata Maxim.

Castanea mollissima Blume

Celastrus orbiculatus Thunb.

Cephalotaxus sinensis (Rehder \& E. H. Wilson) H.

L. Li

Chenopodium album L., also C. giganteum D. Don

Chrysosplenium biondianum Engl.

Cirsium arvense var. setosum (Willd.) C. A. Mey

Cirsium spp. eg Cirsium botryoides Petrak ex

Hand.-Mzt.

Clerodendrum trichotomum Thunb.

Commelina benghalensis L.

Cornus kousa Bürger ex Miq. in Caprifoliaceae)

Amaranthaceae

Actinidiaceae

Campanulaceae

Lardizabalaceae

Amaryllidaceae

(formerly in Liliaceae)

Amaryllidaceae

(formerly in Liliaceae)

Amaryllidaceae

(formerly in Liliaceae)

Amaranthaceae

Amaranthaceae

Asteraceae

Asteraceae

Araliaceae

Asteraceae

Asteraceae

Aristolochiaceae

Aristolochiaceae

Begoniaceae

Rhamnaceae

Urticaceae

Urticaceae

Moraceae

Asteraceae

Brassicaceae

Brassicaceae

Brassicaceae

Lamiaceae (formerly

in Verbenaceae)

Fagaceae

Celastraceae

Cephalotaxaceae

Amaranthaceae

(formerly

Chenopodiaceae)

Saxifragaceae

Asteraceae

Asteraceae

Lamiaceae

Commelinaceae

Cornaceae aerial parts

aerial parts

aerial parts

aerial parts

tender buds

leaf stalks,

underground parts

aerial parts

whole plant (as

spice)

whole plant (as

spice)

aerial parts

fruit

aerial parts

aerial parts

leaves

aerial parts

aerial parts

aerial parts

aerial parts

aerial parts

fruit

aerial parts

pseudo-fruit

aerial parts

aerial parts

aerial parts

aerial parts

aerial parts

aerial parts

fruit

$\begin{array}{cll}* * * & \text { shen xian dou fu } & \text { 神仙豆腐 } \\ * & \text { niu xi } & \text { 牛膝 } \\ * * * & \text { ye mi hou tao } & \text { 野狝猴桃 } \\ * * * & \text { nai jiang cai } & \text { 奶浆菜 }\end{array}$

**** ba yue gua, ba yue zha

* ge jiu, ye jiu

奶浆菜, 八月炸

落非, 野非

$* * * \quad$ tian suan

天蒜

ai jiu cai, ai suan, ye 崖韭菜,

suan, yong bao tou, luo 崖蒜, 野蒜,

er jiu, zong bao tou, ye 罗儿非,

jiu cai

棕包头, 野韭菜

甜菥营

汉菜, 人汉菜,

$* * * *$ han cai, ren han cai

鼠曲草

清明菜

刺龙袍

牛蒡子

艾

毛细辛

细辛

一口血

亚古藤、勾

儿茶

红河麻

河麻

构树果, 构 叶

熊耳朵

地地菜

石夹菜

小石夹菜

臭老汉/老汉香

chou lao han, lao han

xiang

** ye mao li, ye ban li

野毛栗, 野板栗

白蔓叶

白盖果、篦子

树、水柏、松果

灰灰菜

hui hui cai

$* * * \quad$ hong jin cai

红筋菜

** hong hua miao, ci ji

红花苗, 刺蓟

* xiaoji

小蓟

* chou mu dan, chou

臭牡丹、臭

lao han

老汉

* dan zhu ye, zhu ye cao,

mian da zi

淡竹叶、竹叶

草、面达子

*** shi zao 


\begin{tabular}{|c|c|c|c|c|c|}
\hline Scientific name & $\begin{array}{l}\text { Family (according to } \\
\text { APGIII }[31] \text { ) }\end{array}$ & Part used & Frequency & Local name in pinyin & $\begin{array}{l}\text { Local name in } \\
\text { Chinese }\end{array}$ \\
\hline Corylus heterophylla Fisch. ex Besser & Corylaceae & fruit & * & $\begin{array}{l}\text { zhen zi, mao li, mao li } \\
\text { zi shu, xiao xiang zi shu }\end{array}$ & \\
\hline Crataegus hupehensis Sarg. & Rosaceae & fruit & * & ye shan zha & 野山楂 \\
\hline Cryptotaenia japonica Hassk. & Apiaceae & aerial parts & * & ya jiao ban & 鸭脚板 \\
\hline Cynanchum giraldii Schltr. & Asclepiadiaceae & underground parts & * & ge shan xiao & 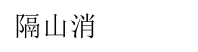 \\
\hline Decaisnea fargesii Franch. & Lardizabalaceae & fruit & $* * *$ & $\begin{array}{l}\text { mao shi gua, ye xiang } \\
\text { jiao }\end{array}$ & 猫屎瓜, 野香蕉 \\
\hline Descurainia sophia (L.) Webb ex Prantl (?) & Brassicaceae & aerial parts & ** & yin chen, mi hao & 因陈, 米蒿 \\
\hline Dioscorea batatas Decne. & Dioscoreaceae & underground parts & * & shan yao & 山药 \\
\hline Elaeagnus umbellata Thunb. & Eleagnaceae & fruit & $* * *$ & yang nai zi, niu nai zi & 羊奶子, 牛奶子 \\
\hline $\begin{array}{l}\text { Fragaria spp. (F. corymbosa Losinsk., F. pentaphylla } \\
\text { Losinsk.) }\end{array}$ & Rosaceae & fruit & $* * *$ & $\begin{array}{l}\text { cao mei, di pao, di di } \\
\text { pao xiang pao }\end{array}$ & $\begin{array}{l}\text { 草莓, 地泡, } \\
\text { 地地泡, 香泡 }\end{array}$ \\
\hline Helwingia japonica (Thunb.) F. Dietr. & Helwingiaceae & leaves & $* * * *$ & ye shang hua & 叶上花 \\
\hline $\begin{array}{l}\text { Hemerocallis spp. (H. dumortierii C. Morren, } H \text {. } \\
\text { fulva L.) }\end{array}$ & $\begin{array}{l}\text { Xanthorrhoeaceae } \\
\text { (formerly Liliaceae) }\end{array}$ & flowers & $* *$ & ye huang hua & 野黄花 \\
\hline Hieracium sp. & Asteraceae & aerial parts & *? & ku mai cai & 苦荬菜 \\
\hline Impatiens notolopha Maxim. & Balsaminaceae & aerial parts & * & dao laon nen & 到老嫩 \\
\hline Ixeris sonchifolia Hance & Asteraceae & aerial parts & $* * *$ & ku mai cai & 苦实菜 \\
\hline Juglans cathayensis Dode & Juglandaceae & fruit & $\star *$ & ye he tao & 野核桃 \\
\hline Kalimeris pinnatifida (Maxim.) Kitam. & Asteraceae & aerial parts & * & ma lan tou & 马兰头 \\
\hline Kochia scoparia (L.) Schrad. & $\begin{array}{l}\text { Amaranthaceae } \\
\text { (formerly } \\
\text { Chenopodiaceae) }\end{array}$ & aerial parts & * & tie sao ba & 铁扫把 \\
\hline Lactuca serriola $\mathrm{L}$. & Asteraceae & aerial parts & $* *$ & $\begin{array}{l}\text { xiao bai jiang, xiao ku } \\
\text { ma cai, ku ma cai }\end{array}$ & $\begin{array}{l}\text { 小苦荬菜, 苦 } \\
\text { 荬菜 }\end{array}$ \\
\hline Leontopodium japonicum Miq. & Asteraceae & aerial parts & * & shi qu cao & 石曲草 \\
\hline Ligusticum sinense Oliv., Chuanhsiung & Apiaceae & aerial parts & $*$ & chuan xiong & 川芦 \\
\hline Lilium giganteum Wall. & Liliaceae & underground parts & ** & shui bai he & 水百合 \\
\hline $\begin{array}{l}\text { Lilium spp. (L. leichtlinii Hook. f., L. tigrinum Ker } \\
\text { Gawl.) }\end{array}$ & Liliaceae & underground parts & ** & ye bai he & 野百合 \\
\hline Lonicera standishii Carr. & Caprifoliaceae & fruit & * & ku tang pao & 苦糖泡 \\
\hline Lychnis senno Siebold \& Zucc. & Caryophyllaceae & whole plant & * & huang hua cai & 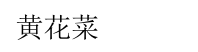 \\
\hline Lycopus lucidus Turcz. ex Benth. & Lamiaceae & aerial parts & * & ye bai cai, ze lan & 野白菜、泽兰 \\
\hline Matteucia struthiopteris (L.) Tod. & Onocleaceae & tender shoots & $* * * *$ & ji tou cai & 鸡头菜 \\
\hline Medicago sativa $\mathrm{L}$. & Fabaceae & aerial parts & * & mu xi cai & 苜蕧菜 \\
\hline Morus australis Poir. & Moraceae & fruit & * & sang pao, sang shu & 桑泡、桑树 \\
\hline $\begin{array}{l}\text { Oxalis spp. (O. griffithii Edgew. \& Hook. f., O. } \\
\text { corniculata L.) }\end{array}$ & Oxalidaceae & aerial parts & * & $\begin{array}{l}\text { suan cao, suan cai, suan } \\
\text { suan cao }\end{array}$ & $\begin{array}{l}\text { 酸草、酸菜、 } \\
\text { 酸酸草 }\end{array}$ \\
\hline Picris hieracioides $\mathrm{L}$. & Asteraceae & aerial parts & *? & ku mai cai & 苦实菜 \\
\hline Pilea mongolica Wedd. & Urticaceae & aerial parts & * & dao lao nen & 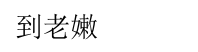 \\
\hline $\begin{array}{l}\text { Pimpinella sp. (probably a new taxon related to } \\
\text { Pimpinella arguta Diels and P. rhomboidea Diels) }\end{array}$ & Apiaceae & aerial parts & $* * * *$ & shui qin cai, sha qin cai & 水芹菜, 沙芹菜 \\
\hline Plantago asiatica $\mathrm{L}$. & Plantaginaceae & aerial parts & * & $\begin{array}{l}\text { kai men ye, che qian } \\
\text { cao, che qian zi }\end{array}$ & $\begin{array}{l}\text { 开门叶、车前 } \\
\text { 草、车前子 }\end{array}$ \\
\hline $\begin{array}{l}\text { Polygonatum megaphyllum P. Y. Li and } \\
\text { Polygonatum odoratum L. }\end{array}$ & $\begin{array}{l}\text { Asparagaceae } \\
\text { (formerly in Liliaceae) }\end{array}$ & underground parts & * & yu zhu, yu zhu shen & 玉竹, 玉竹参 \\
\hline Polygonum ciliinerve (Nakai) Ohwi & Polygonaceae & underground parts & * & qiao mai tou & 菾麦头 \\
\hline $\begin{array}{l}\text { Potentilla arbuscula D. Don var. veitchii ( E. H. } \\
\text { Wilson) Liou }\end{array}$ & Rosaceae & aerial parts & * & guan yin cha & 观音茶 \\
\hline Prunus armeniaca $\mathrm{L}$. & Rosaceae & fruit & $* *$ & ye xing & 野杏 \\
\hline Prunus canescens Bois, P. pilosiuscula Koehne & Rosaceae & fruit & ** & ye ying tao & 野樱桃 \\
\hline Prunus cf. polytricha Koehne & Rosaceae & fruit & * & chuan tao & 川桃 \\
\hline Prunus persica (L.) Batsch & Rosaceae & fruit & $* *$ & ye tao zi & 野桃子 \\
\hline Prunus salicina Lindl. & Rosaceae & fruit & $* * *$ & $\begin{array}{l}\text { ye li zi, ze maili, huo li } \\
\text { zi, huo li, ye mai li }\end{array}$ & $\begin{array}{l}\text { 野李子, } \\
\text { 火李子, 火李, } \\
\text { 野麦李 }\end{array}$ \\
\hline Pteridium aquilinum (L.) Kuhn & Dennstaedtiaceae & $\begin{array}{l}\text { tender shoots and } \\
\text { underground parts }\end{array}$ & $* * * *$ & $\begin{array}{l}\text { jue cai, jue gen, long } \\
\text { zhua }\end{array}$ & $\begin{array}{l}\text { 蕨菜, 蕨根, } \\
\text { 龙爪菜 }\end{array}$ \\
\hline
\end{tabular}




\begin{tabular}{|c|c|c|c|c|c|}
\hline Scientific name & $\begin{array}{l}\text { Family (according to } \\
\text { APGIII [31]) }\end{array}$ & Part used & Frequency & Local name in pinyin & $\begin{array}{l}\text { Local name in } \\
\text { Chinese }\end{array}$ \\
\hline Pueraria lobata (Willd.) Ohwi & Fabaceae & underground parts & $* *$ & ge gen & 葛根 \\
\hline Pyrola decorata Andres & Ericaceae & $\begin{array}{l}\text { aerial parts (spice } \\
\text { and infusion) }\end{array}$ & * & hong ru, shou cha & 红茹、寿茶 \\
\hline Pyrola rotundifolia $\mathrm{L}$. & Ericaceae & $\begin{array}{l}\text { aerial parts (spice } \\
\text { and infusion) }\end{array}$ & * & bairu, shou cha & 白茹、寿茶 \\
\hline Pyrus xerophila T. T. Yu & Rosaceae & fruit & $* * *$ & ye li, ma li, shan li & $\begin{array}{l}\text { 野梨, 麻梨, } \\
\text { 山梨 }\end{array}$ \\
\hline Rhus verniciflua Stokes & Anacardiaceae & aerial parts & * & qi shu & 漆树 \\
\hline Robinia pseudoacacia L. & Fabaceae & flowers & * & huai hua & 槐花 \\
\hline Rorippa montana Small & Brassicaceae & aerial parts & * & man jing cai, la la cai & 蔓茎菜, 辣辣菜 \\
\hline Rosa sp. & Rosaceae & young shoots & * & ci mei hua & 刺玫花 ～～～～～ \\
\hline Rubus coreanus Miq. & Rosaceae & fruit & $* *$ & ci pao, di pao, fu pen zi & $\begin{array}{l}\text { 刺泡, 地泡、 } \\
\text { 覆盆子 }\end{array}$ \\
\hline Rubus flosculosus Focke & Rosaceae & fruit & $* *$ & cai zi pao & 菜子泡 \\
\hline Rubus pungens Cambess. & Rosaceae & fruit & $* *$ & huang ci pao & 黄刺泡 \\
\hline Rubus spp. & Rosaceae & fruit & $* * *$ & $\begin{array}{l}\text { duan yang pao, xuan } \\
\text { gou zi }\end{array}$ & 端阳泡, 悬钩子 \\
\hline Rumex crispus L. & Polygonaceae & aerial parts & $* *$ & $\begin{array}{l}\text { niu she tou, ye da } \\
\text { huang }\end{array}$ & 牛舌头 \\
\hline Sabia shensiensis H. Y. Chen & Sabiaceae & aerial parts & * & $\begin{array}{l}\text { qing teng cai, teng } \\
\text { er cai }\end{array}$ & 青藤菜, 藤儿菜 \\
\hline Saussurea dolichopoda Diels & Asteraceae & aerial parts & $* * *$ & $\begin{array}{l}\text { kong tong cai, kong } \\
\text { xin cai }\end{array}$ & 空筒菜, 空心菜 \\
\hline Schisandra sphenanthera Rehder \& E. H. Wilson & Schisandraceae & fruit & $* * * *$ & wu wei zi & 五味子 \\
\hline $\begin{array}{l}\text { Sedum aizoon L., S. sarmentosum Bunge, } S . \\
\text { pampaninii Raym.-Hamet, S. lineare Thunb. }\end{array}$ & Crassulaceae & aerial parts & $* *$ & $\begin{array}{l}\text { gou ya ban, gou za cai, } \\
\text { machijie, da bu si, chui } \\
\text { pen cao }\end{array}$ & 狗牙瓣, 打不死 \\
\hline Sedum amplibracteatum K. T. Fu & Crassulaceae & aerial parts & $* * *$ & $\begin{array}{l}\text { hua qiao man, la zi } \\
\text { miao, la jiao miao, ye } \\
\text { la cai }\end{array}$ & $\begin{array}{l}\text { 花菾蔓, 野辣 } \\
\text { 子苗苗, } \\
\text { 辣椒苗, 叶辣菜 }\end{array}$ \\
\hline Silene conoidea $\mathrm{L}$. & Caryophyllaceae & aerial parts & * & mai pian cai & 麦片菜 \\
\hline Sinacalia tangutica (Maxim.) B. Nord. & Asteraceae & underground parts & * & shui luo bo & 水萝卜 \\
\hline $\begin{array}{l}\text { Smilacina japonica A. Gray, Smilacina henryi } \\
\text { (Baker) Hara }\end{array}$ & $\begin{array}{l}\text { Asparagaceae } \\
\text { (formerly in Liliaceae) }\end{array}$ & aerial parts & * & pian tou cai & 偏头菜 \\
\hline Stachys affinis Bunge & Lamiaceae & underground parts & * & di gu niu & 地牯牛 \\
\hline Staphylea bumalda DC., S. holocarpa Hemsl. & Staphyleaceae & aerial parts, flowers & $* * * *$ & shu hua cai & 树花菜 \\
\hline Stellaria media (L.) Vill. & Caryophyllaceae & aerial parts & * & e er chang & 我鸟儿肠 \\
\hline Taraxacum mongolicum Han.-Mzt & Asteraceae & aerial parts & $* *$ & $\begin{array}{l}\text { pu gong ying, ku mai } \\
\text { cai, da ku cai }\end{array}$ & $\begin{array}{l}\text { 蒲公英、苦荬 } \\
\text { 菜、大苦菜 }\end{array}$ \\
\hline Thlaspi arvense $\mathrm{L}$. & Brassicaceae & aerial parts & $* * *$ & ji dan huang & 鸡蛋黄 \\
\hline Toona sinensis (Juss.) M. Roem. & Meliaceae & tender leaf shoots & $* * * *$ & xiang chun & 香椿 \\
\hline Tricyrtis macropoda Miq. & Liliaceae & aerial parts & $* * *$ & huang gua cai & 黄瓜菜 \\
\hline $\begin{array}{l}\text { Ulmus bergmanniana C. K. Schneid., U. propinqua } \\
\text { Koidz., U. pumila L. }\end{array}$ & Ulmaceae & $\begin{array}{l}\text { leaves, bark, } \\
\text { immature fruit }\end{array}$ & ** & yu shu & 榆树 \\
\hline Urtica fissa E. Pritz. ex Diels & Urticaceae & aerial parts & * & bai he ma & 白河麻 \\
\hline Vicia cracca L. & Fabaceae & aerial parts & * & ye wan dou jian & 野豌豆尖 \\
\hline Vitis ficifolia Bunge & Vitaceae & fruit & $* * *$ & ye pu tao & 野葡萄 \\
\hline Zanthoxylum bungeanum Maxim. & Rutaceae & fruits, aerial parts & * & ye hua jiao & 野花椒 \\
\hline \multicolumn{6}{|l|}{ Fungi } \\
\hline Boletus spp. & Boletaceae & & $* *$ & niu gan jun, da jiao gu & 牛肝菌, 大脚菇 \\
\hline Cantharellus cibarius Fr. & Cantharellaceae & & $* * *$ & huang si jun & 牛肝菌 \\
\hline Grifola umbellata (Pers.) Pilát & Meripilaceae & & $* * *$ & zhu ling jun & 猪苓菌 \\
\hline Hericium sp. & Hericiaceae & & $* *$ & hou tou jun & 猴头菌 \\
\hline Laetiporus sulphureus (Bull.) Murrill (??) & Polyporaceae & & * & ji guan jun & 鸡冠菌 \\
\hline $\begin{array}{l}\text { Lentinula edodes (Berk.) Pegler (more often } \\
\text { cultivated) }\end{array}$ & Marasmiaceae & & * & ye xiang gu & 野香菇 \\
\hline Morchella sp. & Morchellaceae & & $* *$ & yang que jun & 羊雀菌 \\
\hline
\end{tabular}




\begin{tabular}{|c|c|c|c|c|c|}
\hline Scientific name & $\begin{array}{c}\text { Family (according to } \\
\text { APGIII [31]) }\end{array}$ & Part used & Frequency & Local name in pinyin & $\begin{array}{l}\text { Local name in } \\
\text { Chinese }\end{array}$ \\
\hline Pleurotus sp. & Pleurotaceae & & * & dong jun & 冻菌 \\
\hline Ramaria spp. & Gomphaceae & & $* *$ & shua ba jun & 刷把菌 \\
\hline Unidentified terrestrial gilled mushroom & $?$ & & ** & ban li jun & 板栗菌 \\
\hline Unidentified mushroom & $?$ & & * & bao gu jun & 包谷菌 \\
\hline Unidentified mushroom & $?$ & & * & qiao mian jun & 菾面菌 \\
\hline
\end{tabular}

Frequency: ${ }^{\star \star \star *}>50 \%$ of respondents; ${ }^{* \star *}>1 / 4$ of respondents; ${ }^{\star \star}>1 / 8$ of respondents; ${ }^{\star} 1 / 8$ of respondents or less, but at least 2 respondents.

Pteridium aquilinum, Polygonatum spp., Sinacalia tangutica, the bulbs of Lilium giganteum and other Lilium species, and the bark of Ulmus spp. Nowadays the consumption of underground organs of plants has practically ceased and is restricted to the occasional use of Lilium spp. and Stachys affinis.

Wild vegetables are eaten in any of the daily three meals. The commonest preparation technique is boiling, then straining and sprinkling them with some oil in which Sechuan pepper, garlic, and sometimes ginger, was fried. Then they are served, warm or cold. This is a side dish, called "liang ban", accompanied by home-made wheat bread ("bing"), rice or other stirfried foods. Sometimes wild vegetables are also put into broad, home-made noodles served in spicy and sour soup. They are also, rarely, lacto-fermented. Dried vegetables are first soaked in water for a few minutes or hours and then used like fresh vegetables. Some respondents say that in the case of Staphylea shoots, drying even improves their taste and digestibility. Wild vegetables are also sold in all the local restaurants (Tab. 2), and every agritourist farm has them on their menu.

Fruits are and have always been less appreciated than wild greens. They are sometimes collected for fun by children or grown-ups going to the forest to collect wild greens, medicinal herbs or wood. They have never been stored for winter and are not used in any dishes by anyone, apart from dried Schisandra and Akebia fruits, used medicinally. In spite of this, most people easily mentioned a few species of wild fruits they had eaten.

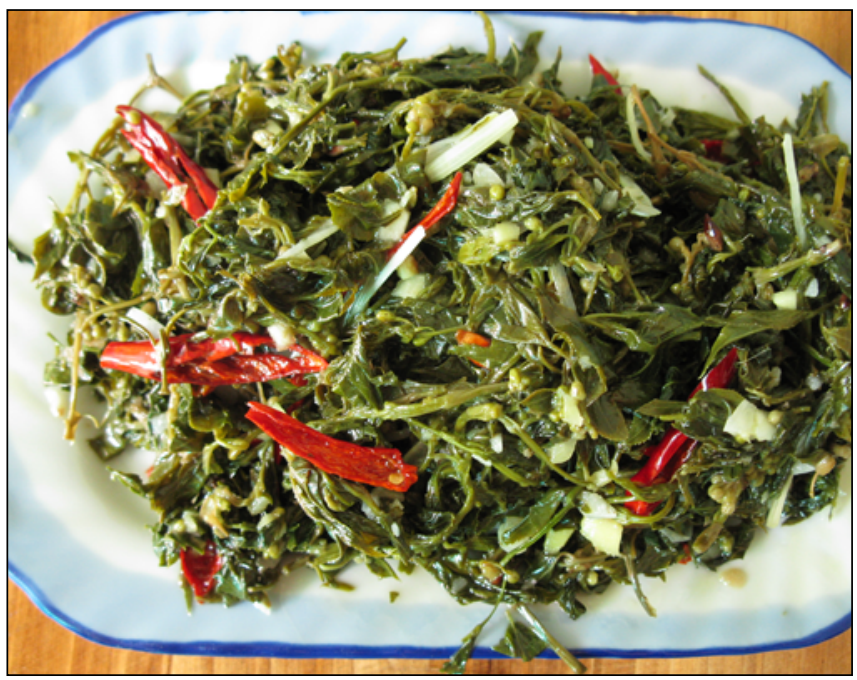

Fig. 2 Staphylea bumalda flowering shoots, boiled, strained and sprinkled with oil, Houzhenzi, 2011. Photograph by Łukasz Łuczaj.
Few fungi species are used, as most of them are generally feared. We found only one inhabitant of the valley, who had a hobby of collecting wild edible mushrooms, which he developed after gathering Boletus species for sale a few years ago. Others never go to the forest with the purpose of collecting mushrooms, apart from going to collect cultivated Auricularia and Lentinula edodes grown on piles of logs located in the woods. The only mushrooms relatively more widely known and accepted are Cantharellus cibarius, an unidentified Agaricales (called "banlijun", i.e. "chestnut mushroom"), Ramaria spp. (and possibly Clavaria spp.) treated by locals as one folk taxon "shuabajun" (i.e. "brush mushroom") and Grifola umbellata, whose sclerotia are collected for medicinal purposes, although fruiting bodies are occasionally eaten as well. However, only a third of the respondents have ever eaten the most commonly listed mushroom, Cantharellus cibarius. More than half of the respondents had never collected wild fungi in the forest. A few people who eat Boletus spp. species started doing so after taking part in commercial mushroom collecting a few years ago.

\section{Discussion}

The large number of used wild greens in this study is one of the highest recorded on such a small scale in the history of ethnobotanical studies. The only comparable study, by Zou [15], recorded the use of 335 taxa of wild vegetables, belonging to 87

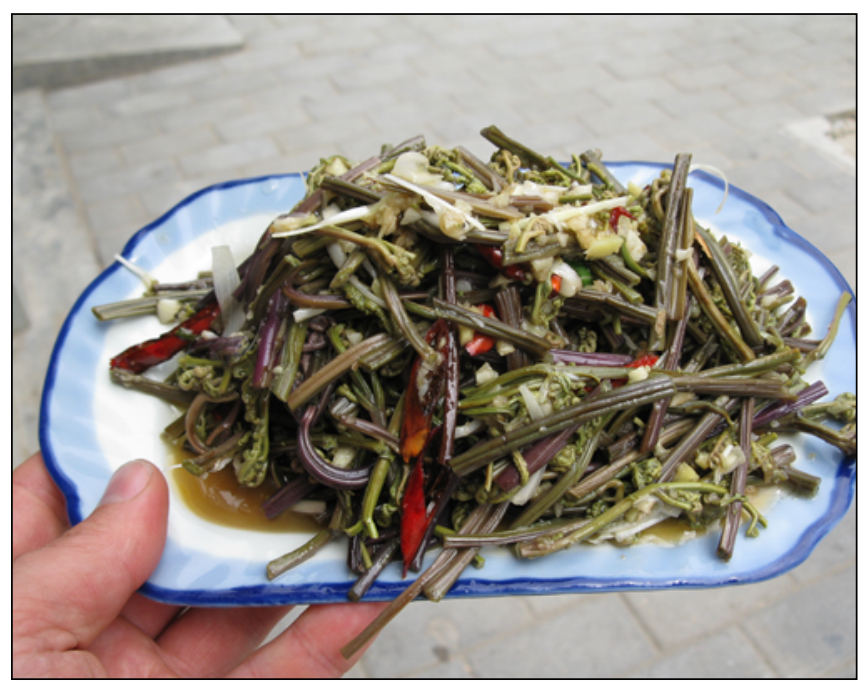

Fig. 3 Pteridium aquilinum shoots, boiled, strained and sprinkled with oil, Houzhenzi, 2011. Photograph by Łukasz Łuczaj. 


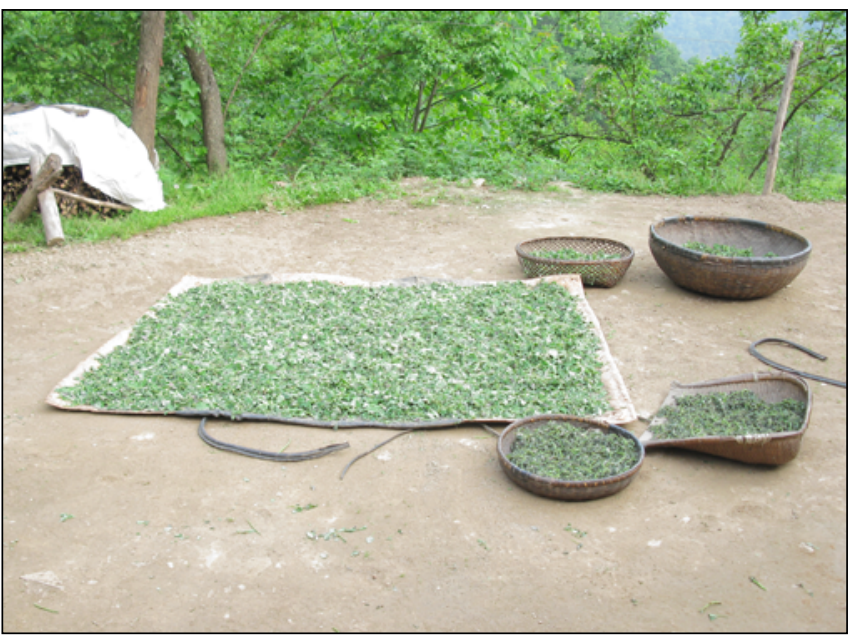

Fig. 4 Drying wild vegetables (Chenopodium album and Staphylea bumalda) on a farm in Diaoyutai in early June 2011. Photograph by Łukasz Łuczaj.

families and 119 genera in 10 villages of Hunan, however the latter study concerned a larger and more heterogenous area. Ghorbani [16] recorded the use of 173 wild food plants from 485 informants of four ethnic groups of Xishuanbanna valley, out of which only around a third were wild greens. However, his study concerned an area which was very heterogenous in terms of elevation, inhabitants and vegetation. The average number of wild food plants listed by one informant was only around 10 species, whereas in this study we documented a much higher rate of use per person (mean - over 24 species), with probably the highest average number of wild green vegetables listed per person (mean - 17 species) using freelisting techniques in any ethnobotanical study to date.

Knowledge of wild vegetables in China is additionally encoded in the language. Most wild vegetables have the word "cai", i.e. vegetable, so it is enough to know the name and to be able to recognize the plant to be able to presume its use, i.e. if it is a "cai" (vegetable) or "cao"/“yao" (medicinal plant).

The relatively high geographical diversity of the use of "ye cai" in China should be emphasized. For example none of the articles on wild vegetables of Qinling Mts mention the use of Staphylea spp. nor Pimpinella sp. - locally important vegetables $[19,20]$.

The widespread phenomenon of drying wild vegetables is worth attention. This ancient preservation technique is nowadays rarely used for wild vegetables across the globe. Storing a particular food for winter may mean that this is a culturally significant item.

Several taxa are semi-domesticated, and undergo varying degrees of active protection. Some are increasingly brought from the forest to be planted in gardens (Toona sinensis, Staphylea bumalda, Asarum sieboldii). The two species of mushrooms widely cultivated using tree logs left in the garden or in the forest, Auricularia sp. and Lentinula edodes, were mentioned by some respondents as wild vegetables as well. This shows that, similarly to other geographic areas, the distinction between the wild and the cultivated is not usually sharp $[2,25,26]$.

The local population utilizes a large proportion of the local edible flora. However, strong cultural biases can be seen even in such a herbophilous community. Some edible wild vegetables, e.g. Galinsoga sp., Reynoutria japonica, most Polygonum species and Lamium barbatum are not used, the common Stellaria media, is also eaten rarely and only by a few individuals.

Over half wild vegetables come from the forest. This is in contrast with some studies showing that human populations, even in wooded areas, tend to over-utilize the ruderal flora $[27,28]$. Here, at least two explanations are possible:

(i) the fields' area is relatively small, so in the past the volume of wild vegetables from ruderal sites may not have been sufficient,

(ii) many edible ruderal taxa are regarded here as pig food, and we observed some degree of separating wild vegetables for human consumption and pig food (the exception here is Chenopodium, eaten both by humans and pigs).

What is interesting is the large domination of wild greens over fruits and fungi. A typical answer of a respondent to the question about what wild veg they had eaten was: "ye cai hen duo" (there are many wild vegetables). On the other hand, the same question about fruits or fungi resulted in the opposite answer, e.g. "ye mogu/junzi hen shao" (there are very few wild fungi).

Wild vegetables are intentionally collected here, they are well known to everyone and dried for winter in most houses. Fruits are something unimportant, play objects, something one finds on a forest walk to collect herbs, something only eaten raw. They are never cooked or dried or added to any dishes. The only exceptions are the fruits of Schisandra, which are dried and sold as medicine. The lack of interest in mushrooms is puzzling, as China is usually regarded as a mycophilous part of the world $[3,29,30]$.

\section{Conclusions}

The studied community displays one of the highest levels of herbophilia known in human cultures. On the other hand the community shows relative indifference to wild fruits and fungi, which are rarely collected, and only as an additional activity.

The results of this study show that further in-depth ethnobotanical research is needed to determine patterns in wild food plant and fungi use in different parts of China, as locally these patterns may be extremely variable.

\section{Acknowledgments}

Many thanks to the inhabitants of the studied villages for their generous help in sharing information on the use of the species. The program was financially supported by the Forestry Research Foundation for the Public Service Industry of China (2009,04004) and by the University of Rzeszów (Institute of Biotechnology and Basic Sciences, as well as a special grant from the rector of the University). We are also grateful to Prof. Khasbagen (Hohhot, China) for his help in literature search.

\section{References}

1. Łuczaj $Ł$. Changes in the utilization of wild green vegetables in Poland since the 19th century: a comparison of four ethnobotanical surveys. J Ethnopharmacol. 2010;128(2):395-404. http://dx.doi.org/10.1016/j. jep.2010.01.038 
2. Turner NJ, Łuczaj ŁJ, Migliorini P, Pieroni A, Dreon AL, Sacchetti LE, et al. Edible and tended wild plants, traditional ecological knowledge and agroecology. Crit Rev Plant Sci. 2011;30(1-2):198-225. http://dx.doi.org /10.1080/07352689.2011.554492

3. Boa ER. Wild edible fungi: a global overview of their use and importance to people. Rome: FAO; 2004.

4. Anderson EN. The food of China. New Haven: Yale University Press; 1988.

5. Hu SY. Food plants of China. Hong Kong: Chinese University Press; 2005.

6. Long CL. Strategies for agrobiodiversity conservation and promotion: a case from Yunnan, China. Biodivers Conserv. 2003;12(6):1145-1156.

7. Wu X. "Turning waste into things of value": marketing fern, Kudzu, and Osmunda in Enshi Prefecture, China. J Dev Soc. 2003;19(4):433-457. http://dx.doi.org/10.1177/0169796X0301900401

8. Wang X, Du X. Recent status of the development and strategies of exploitation of non wood forest products in China. Lin Ye Ke Xue Yan Jiu. 1997;10(2):199-205.

9. You-Kai X, Guo-Da T, Hong-Mao L, Kang-La Y, Xiang-Sheng D. Wild vegetable resources and market survey in Xishuangbanna, southwest China. Econ Bot. 2004;58(4):647-667. http://dx.doi. org/10.1663/0013-0001(2004)058[0647:WVRAMS]2.0.CO;2

10. Zhang XP, Wu JL, Li Y, Liu F, Wang JQ. Investigation on species resources and utilization of wild vegetable in nabanhe watershed nature reserve, Xishuangbanna. Journal of Southwest Forestry College. 2004;24:21-24.

11. Huai KHY, Pei SJ. Wild plants in the diet of Arhorchin Mongol herdsmen in inner Mongolia. Econ Bot. 2000;54(4):528-536. http://dx.doi. org/10.1007/BF02866550

12. Wujisguleng $\mathrm{W}$, Khasbagen $\mathrm{K}$. An integrated assessment of wild vegetable resources in Inner Mongolian Autonomous Region, China. J Ethnobiol Ethnomed. 2010;6(1):34. http://dx.doi.org/10.1186/1746-4269-6-34

13. Weckerle CS, Huber FK, Yongping Y, Weibang S. Plant knowledge of the Shuhi in the Hengduan mountains, Southwest china. Econ Bot. 2006;60(1):3-23. http://dx.doi. org/10.1663/0013-0001(2006)60[3:PKOTSI]2.0.CO;2

14. Huber FK, Ineichen R, Yang Y, Weckerle CS. Livelihood and conservation aspects of non-wood forest product collection in the Shaxi Valley, southwest China. Econ Bot. 2010;64(3):189-204. http://dx.doi.org/10.1007/ s12231-010-9126-Z

15. Zou X, Huang F, Hao L, Zhao J, Mao H, Zhang J, et al. The socioeconomic importance of wild vegetable resources and their conservation: a case study from China. Kew Bull. 2011;65(4):577-582. http://dx.doi. org/10.1007/s12225-010-9239-7

16. Ghorbani A, Langenberger G, Sauerborn J. A comparison of the wild food plant use knowledge of ethnic minorities in Naban River Watershed National Nature Reserve, Yunnan, SW China. J Ethnobiol Ethnomed. 2012;8(1):17. http://dx.doi.org/10.1186/1746-4269-8-17

17. Ghorbani A, Langenberger G, Liu JX, Wehner S, Sauerborn J. Diversity of medicinal and food plants as non-timber forest products in Naban River
Watershed National Nature Reserve (China): implications for livelihood improvement and biodiversity conservation. Econ Bot. 2012;66(2):178191. http://dx.doi.org/10.1007/s12231-012-9188-1

18. Jin C, Yin-Chun S, Gui-Qin C, Wen-Dun W. Ethnobotanical studies on wild edible fruits in southern Yunnan: folk names; nutritional value and uses. Econ Bot. 1999;53(1):2-14. http://dx.doi.org/10.1007/BF02860785

19. Ma X, Zhang J, Lu S, Cui Z, Zhao H, Zheng J. The survey of the distribtion of wild vegetables in the northern slope of the Qinling Mountains. Quaterly of Forest By-Product and Speciality in China. 2002;61(2):49-50.

20. Ma X, Hu P, Wu J, Zhang J, Cui Z. The habitat, development and utilization of wild vegetables in the northern slope of the Qinling Mountains. Special Economic Animal. 2002;2:37-38.

21. Economic survey of the Zhouzhi County Houzhenzi town, protected areas from 2007 [Internet]. 2007 [cited 2012 Sep 20]; Available from: http://wwf. nwsuaf.edu.cn/article/2007/0912/news_219.html

22. Kang Y, Łuczaj ŁJ, Ye S. The highly toxic Aconitum carmichaelii Debeaux as a root vegetable in the Qinling Mountains (Shaanxi, China). Genet Resour Crop Evol. 2012;59(7):1569-1575. http://dx.doi.org/10.1007/ s10722-012-9853-3

23. Quinlan M. Considerations for collecting freelists in the field: examples from ethobotany. Field Methods. 2005;17(3):219-234. http://dx.doi. org/10.1177/1525822X05277460

24. Sutrop U. List task and a cognitive salience index. Field Methods. 2001;13(3):263-276. http://dx.doi.org/10.1177/1525822X0101300303

25. McClatchey WC. Wild food plants of Remote Oceania. Acta Soc Bot Pol. 2012;81(4):371-380. http://dx.doi.org/10.5586/asbp.2012.034

26. Łuczaj Ł, Pieroni A, Tardío J, Pardo-de-Santayana M, Sõukand R, Svanberg I, et al. Wild food plant use in 21st century Europe: the disappearance of old traditions and the search for new cuisines involving wild edibles. Acta Soc Bot Pol. 2012;81(4):359-370. http://dx.doi.org/10.5586/asbp.2012.031

27. Voeks RA. Disturbance pharmacopoeias: medicine and myth from the humid tropics. Ann Assoc Am Geogr. 2004;94(4):868-888. http://dx.doi. org/10.1111/j.1467-8306.2004.00439.x

28. Sõukand R, Kalle R. Change in medical plant use in Estonian ethnomedicine: a historical comparison between 1888 and 1994. J Ethnopharmacol. 2011;135(2):251-260. http://dx.doi.org/10.1016/j.jep.2011.02.030

29. Yamin-Pasternak S. Ethnomycology: fungi and mushrooms in cultural entanglements. In: Anderson EN, Pearsall D, Hunn E, Turner N, editors. Ethnobiology. Hoboken NJ: Wiley and Sons; 2011. p. 213-230. http:// dx.doi.org/10.1002/9781118015872.ch13

30. Arora D. The houses that matsutake built. Econ Bot. 2008;62(3):278-290. http://dx.doi.org/10.1007/s12231-008-9048-1

31. The Angiosperm Phylogeny Group. An update of the Angiosperm Phylogeny Group classification for the orders and families of flowering plants: APG III. Bot J Linn Soc. 2009;161(2):105-121. http://dx.doi. $\operatorname{org} / 10.1111 /$ j.1095-8339.2009.00996.x 